Working Papers

Institute of

Mathematical

Economics

\title{
Convex vNM-Stable Sets for Linear Production Games
}

Joachim Rosenmüller and Benyamin Shitovitz

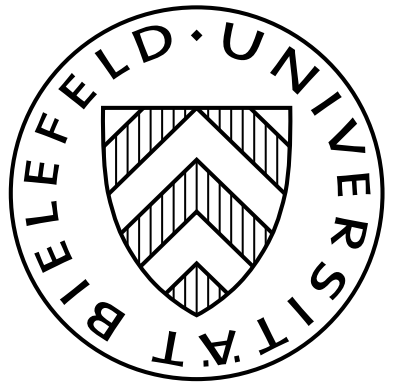




\title{
Convex vNM-Stable Sets for Linear Production Games
}

\author{
Joachim Rosenmüller $^{1}$ and Benyamin Shitovitz ${ }^{2}$ \\ ${ }^{1}$ Institut für Mathematische Wirtschaftsforschung \\ Universität Bielefeld \\ D-33615 Bielefeld, Germany \\ e-mail: jr@wiwi.uni-bielefeld.de \\ ${ }^{2}$ Department of Economics \\ Haifa University \\ Mount Carmel, Haifa 31905, Israel \\ e-mail: binya@econ.haifa.ac.il
}

2008

\begin{abstract}
We characterize convex vNM-Stable Sets according to von Neumann and Morgenstern for orthogonal linear production games with a continuum of players.

The results of [6] are thereby substantially improved. Simultaneously, this is a corrigendum concerning the proof of a lemma in our first paper.
\end{abstract}

*The authors are indebted to Evan Shellshear for having pointed out an error in the previous paper. 


\section{Introduction}

Within this paper we prove a generalization of the main theorem in [6], the Characterization Theorem for convex vNM-Stable Sets of non-atomic orthogonal linear production games. While in [6] we show that every polyhedral vNM-Stable Set is standard, we are now in the position to omit the quantifier "polyhedral". Thus, we show that every convex vNM-Stable Set is standard (i.e., the convex hull of finitely many imputations concentrated on the carriers of the orthogonal measures defining the game). This is certainly good news as we provide a characterization given the weakest possible conditions.

There is also less good news. Unfortunately, this paper also has to serve as a corrigendum. The proof of the Orthogonality Theorem (Theorem 4.8. in [6]) contains an error and cannot be sustained. While the Theorem is true, the approach chosen could not be repaired and a completely different method for the proof of our result had to be supplied.

The proof we are presenting here is more general and yields the desired Characterization Theorem directly. Therefore, we hope that this paper can be regarded as to supply more than just a corrigendum.

We are indebted to Evan Shellshear, IMW, The University of Bielefeld, for having pointed out the error to us. (See also [4]).

\section{Notations and Definitions}

We shortly repeat some of the notations necessary for our presentation. The motivations as well as orientation regarding the existing literature is found in [6].

A game is a triple $(I, \underline{\underline{\mathbf{F}}}, \boldsymbol{v})$, such that $I$ is some interval in the reals (the

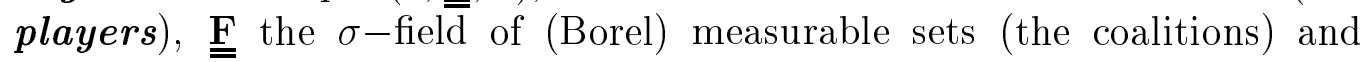
$\boldsymbol{v}: \underline{\underline{\mathbf{F}}} \rightarrow \mathbb{R}_{+}$a function absolutely continuous w.r.t Lebesgue measure $\boldsymbol{\lambda}$ (the coalitional function). We call $\boldsymbol{v}$ the game as well as the basic data will not change. We focus on linear production games generated by finitely many orthogonal measures $\boldsymbol{\lambda}^{1}, \ldots, \boldsymbol{\lambda}^{r}$ via

$$
\boldsymbol{v}(S):=\min \left\{\boldsymbol{\lambda}^{\rho}(S) \mid \rho=1, \ldots, r\right\} \quad(S \in \underline{\underline{\mathbf{F}}}),
$$

which is written

$$
\boldsymbol{v}=\bigwedge\left\{\boldsymbol{\lambda}^{1}, \ldots, \boldsymbol{\lambda}^{r}\right\}
$$

We assume that the $\boldsymbol{\lambda}^{\rho}$ are absolutely continuous with respect to Lebesgue measure (the reference measure), defined on disjoint intervals $\boldsymbol{C}^{\rho}, \quad(\rho=$ $1, \ldots, r)$, the union of which is $\boldsymbol{I}$. 
We use $\bigwedge$ to denote the min operation in the lattice of set functions on $\underline{\underline{\mathbf{F}}}$; equations (2.1) and (2.2) provide the standard usage. The carrier of a measure $\boldsymbol{\mu}$ is denoted by $\boldsymbol{C}(\boldsymbol{\mu})$, in the context of a game given by (2.2)accordingly we use the abbreviation $\boldsymbol{C}^{\rho}:=\boldsymbol{C}\left(\boldsymbol{\lambda}^{\rho}\right)$. Recall the concept of a $\boldsymbol{v} \boldsymbol{N} \boldsymbol{M}$-Stable Set (von Neumann-Morgenstern [7]).

Definition 2.1. Let $(I, \underline{\underline{\mathbf{F}}}, \boldsymbol{v})$ be a game. An imputation is a measure $\boldsymbol{\xi}$ with $\boldsymbol{\xi}(I)=\boldsymbol{v}(I)$. An imputation $\xi$ dominates an imputation $\boldsymbol{\eta}$ w.r.t $S \in \underline{\underline{\mathbf{F}}}$ if $\boldsymbol{\xi}$ is effective for $S$, i.e.,

$$
\boldsymbol{\lambda}(S)>0 \text { and } \boldsymbol{\xi}(S) \leq \boldsymbol{v}(S)
$$

and if

$$
\boldsymbol{\xi}(T)>\boldsymbol{\eta}(T) \quad(T \in \underline{\underline{\mathbf{F}}}, T \subseteq S, \boldsymbol{\lambda}(T)>0)
$$

holds true, that is, every subcoalition of $S$ (almost every player in $S$ ) strictly improves its payoff at $\boldsymbol{\xi}$ versus $\boldsymbol{\eta}$. We write $\boldsymbol{\xi} \operatorname{dom}_{S} \boldsymbol{\eta}$ to indicate domination.

We allow domination also to take place between 'subimputations', i.e., measures with total mass less than $\boldsymbol{v}(I)$.

Definition 2.2. Let $\boldsymbol{v}$ be a game. A set $\mathcal{S}$ of imputations is called a $\boldsymbol{v} \boldsymbol{N} \boldsymbol{M}-$ Stable Set if

- there is no pair $\boldsymbol{\xi}, \boldsymbol{\mu} \in \mathcal{S}$ such that $\boldsymbol{\xi} \operatorname{dom}_{S} \boldsymbol{\mu}$ w.r.t. some coalition $S \in \underline{\underline{\mathbf{F}}}$

- for every imputation $\boldsymbol{\eta} \notin \mathcal{S}$ there exists $\boldsymbol{\xi} \in \mathcal{S}$ such that, for some $S \in \underline{\underline{\mathbf{F}}}$ the relation $\boldsymbol{\xi} \operatorname{dom}_{S} \boldsymbol{\eta}$ is satisfied.

We restrict the discussion to vNM-Stable Sets containing measures only which that are absolutely continuous w.r.t. the "reference measure"

$$
\boldsymbol{\lambda}^{0}:=\sum_{\rho=1}^{r} \boldsymbol{\lambda}^{\rho} .
$$

The assumption of an underlying reference measure and existing densities for the members of a vNM-Stable Set is justified in [6].

A linear production game may be normalized to yield

$$
1=\boldsymbol{v}(I)=\boldsymbol{\lambda}^{1}(I) \leq \boldsymbol{\lambda}^{\rho}(I) \quad(\rho=1, \ldots, r) .
$$

Definition 2.3. Let $\boldsymbol{v}=\bigwedge\left\{\boldsymbol{\lambda}^{1}, \ldots, \boldsymbol{\lambda}^{r}\right\}$ be a normalized orthogonal linear production game and let $\boldsymbol{\mu}^{1}, \ldots, \boldsymbol{\mu}^{r}$ be probabilities such that $\boldsymbol{\mu}^{\rho} \ll \boldsymbol{\lambda}^{\rho}$, $\frac{d \boldsymbol{\mu}^{\rho}}{d \boldsymbol{\lambda}^{\rho}} \leq$ $1, \rho=1, \ldots, r$ holds true. Then the $v N M$-Stable Set $\mathcal{S}=\boldsymbol{C o n v} \boldsymbol{H}\left\{\boldsymbol{\mu}^{1}, \ldots, \boldsymbol{\mu}^{r}\right\}$ is called a standard solution. 


\section{The Main Theorem of Characterization}

The first important result in [6] states that a standard solution is indeed a vNM-Stable Set. The reverse result according to which every vNM-Stable Set is standard is based on several lemmata (named the Density Lemma, the Inheritance Lemma etc.) which we do not repeat here. As we have mentioned above, the proof of the Orthogonality Theorem cannot be sustained in the general case (it is actually correct for two extremals). The following proof replaces the faulty version and extends its result, not assuming the polyhedral shape of the solution. This condition was introduced in [6] in order to provide extremals. As we see below, none of these assumptions is necessary. We just need convexity.

Theorem 3.1. Let $\mathcal{S}$ be a convex $v N M-$ Stable Set. Then $\mathcal{S}$ is standard.

Proof: We fix some element $\boldsymbol{\eta} \in \mathcal{S}$. Within the first 5 steps we show essentially that $\boldsymbol{\eta}$ is a convex combination of certain imputations in $\mathcal{S}$ that are concentrated on the carriers of the generating measures $\boldsymbol{\lambda}^{\rho}(\rho=1, \ldots, r)$. The theorem then follows immediately from a result in [6] (the sixth step below).

In detail we proceed as follows.

$1^{\text {st }}$ STEP :

Let $\boldsymbol{\eta} \in \mathcal{S}$ be an arbitrary element of our solution.

$$
c_{\rho}:=\boldsymbol{\eta}\left(\boldsymbol{C}^{\rho}\right) \quad \rho \in\{1, \ldots, r\}
$$

and let

$$
\begin{gathered}
\boldsymbol{K}_{0}:=\left\{\rho \mid c_{\rho}=0\right\} \subseteq\{1, \ldots r\} \\
\boldsymbol{K}_{+}:=\left\{\sigma \mid c_{\sigma}>0, \frac{\boldsymbol{\eta}_{\mid \boldsymbol{C}^{\sigma}}}{c_{\sigma}} \in \mathcal{S}\right\} \subseteq\{1, \ldots r\} \\
\boldsymbol{K}_{-}:=\left\{\tau \mid c_{\tau}>0, \frac{\boldsymbol{\eta}_{\mid \boldsymbol{C}^{\tau}}}{c_{\tau}} \notin \mathcal{S}\right\} \subseteq\{1, \ldots r\}
\end{gathered}
$$

such that

$$
\sum_{\rho \in \boldsymbol{K}_{+} \cup \boldsymbol{K}_{-}} c_{\rho}=1
$$

holds true.

For $\sigma \in \boldsymbol{K}_{+}$let

$$
\boldsymbol{\vartheta}^{(\sigma)}:=\frac{\boldsymbol{\eta}_{\boldsymbol{I} \boldsymbol{C}^{\sigma}}}{c_{\sigma}} \in \mathcal{S} .
$$

Now, if it turns out that $\boldsymbol{K}_{-}=\emptyset$, then we have

$$
\sum_{\rho \in \boldsymbol{K}_{+}} c_{\rho} \boldsymbol{\vartheta}^{(\rho)}=\sum_{\rho \in \boldsymbol{K}_{+}} \boldsymbol{\eta}_{\mid \boldsymbol{C}^{\rho}}=\boldsymbol{\eta}
$$


that is, $\boldsymbol{\eta}$ is a convex combination of orthogonal elements of $\mathcal{S}$. The Theorem then follows at once as will be explained in the $6^{\text {th }} S T E P$. Therefore, it is our aim to prove the emptyness of $\boldsymbol{K}_{-}$.

\section{$2^{\text {nd }}$ STEP :}

Consequently, we will now continue under the assumption that $\boldsymbol{K}_{-} \neq \emptyset$. This will lead to a contradiction.

Now, for $\tau \in \boldsymbol{K}_{-}$pick $\boldsymbol{\vartheta}^{(\tau)} \in \mathcal{S}$ and $R_{(\tau)}$ such that

$$
\boldsymbol{\vartheta}^{(\tau)} \operatorname{dom}_{R_{(\tau)}} \frac{\boldsymbol{\eta}_{\boldsymbol{I} \boldsymbol{C}^{\tau}}}{c_{\tau}}
$$

That is, for $\tau \in \boldsymbol{K}_{-}$, we have

$$
\boldsymbol{\vartheta}^{(\tau)}>\frac{\boldsymbol{\eta}_{\mathbf{I} \boldsymbol{C}^{\tau}}}{c_{\tau}} \text { on } R_{(\tau)} \text { and } \boldsymbol{\vartheta}^{(\tau)}\left(R_{(\tau)}\right) \leq \boldsymbol{v}\left(R_{(\tau)}\right)
$$

We introduce the notation

$$
R_{(\tau)}=R_{(\tau)}^{1} \cup R_{(\tau)}^{2} \cdots \cup R_{(\tau)}^{r}
$$

where $R_{(\tau)}^{\rho}=R_{(\tau)} \cap \boldsymbol{C}^{\rho}$ for $\rho=1, \ldots, r$ as usual. Then we rewrite (3.2)

$$
c_{\tau} \boldsymbol{\vartheta}^{(\tau)}>\boldsymbol{\eta}_{\mid \boldsymbol{C}^{\tau}} \quad \text { on } R_{(\tau)} \quad\left(\tau \in \boldsymbol{K}_{-}\right)
$$

and

$$
c_{\tau} \boldsymbol{\vartheta}^{(\tau)}\left(R_{(\tau)}\right) \leq c_{\tau} \boldsymbol{v}\left(R_{(\tau)}\right) \quad\left(\tau \in \boldsymbol{K}_{-}\right)
$$

There is no harm in assuming for $\tau \in \boldsymbol{K}_{-}$

$$
\begin{aligned}
\boldsymbol{v}\left(R_{(\tau)}\right) & =\lambda^{1}\left(R_{(\tau)}\right)=\lambda^{1}\left(R_{(\tau)}^{1}\right) \\
& =\lambda^{2}\left(R_{(\tau)}\right)=\lambda^{2}\left(R_{(\tau)}^{2}\right) \\
& =\quad \cdots \\
& =\lambda^{r}\left(R_{(\tau)}\right)=\lambda^{r}\left(R_{(\tau)}^{r}\right)
\end{aligned}
$$

Moreover, by applying Lemma 4.5 (The Inheritance Lemma) in [6], we may decrease some of the $R_{(\tau)}$ such that eventually $\boldsymbol{v}\left(R_{(\tau)}\right)=\boldsymbol{v}\left(R_{\left(\tau^{\prime}\right)}\right)\left(\tau, \tau^{\prime} \in\right.$ $\boldsymbol{K}_{-}$) and hence the quantities in (3.5) coincide for varying $\tau \in \boldsymbol{K}_{-}$. Also, note that

$$
\boldsymbol{\vartheta}^{(\tau)} \text { is positive on } R_{(\tau)}\left(\tau \in \boldsymbol{K}_{-}\right)
$$

\section{$3^{\text {rd }}$ STEP :}

Now we define

$$
\boldsymbol{\vartheta}:=\sum_{\sigma \in \boldsymbol{K}_{+} \cup \boldsymbol{K}_{-}} c_{\sigma} \boldsymbol{\vartheta}^{(\sigma)}
$$


Furthermore, we will define a measurable set $S=S^{1} \cup \ldots \cup S^{r}$ which will serve for domination of $\boldsymbol{\eta}$ eventually.

First of all, for $\tau \in \boldsymbol{K}_{-}$we put

$$
S^{\tau}:=R_{(\tau)}^{\tau}
$$

Next, for $\sigma \in \boldsymbol{K}_{+}$we choose $S^{\sigma} \subseteq \boldsymbol{C}^{\sigma}$ such that $\boldsymbol{\eta}_{\text {। } S^{\sigma}}>0$ and

$$
\lambda^{\sigma}\left(S^{\sigma}\right)=\lambda^{\sigma}\left(S \cap \boldsymbol{C}^{\sigma}\right)=\boldsymbol{v}\left(R_{(\tau)}\right) \quad\left(\tau \in \boldsymbol{K}_{-}\right) .
$$

The common value on the right hand side of (3.8) is the one defined in (3.5), so that now all the quantities in (3.5) and (3.8) again coincide. As $\boldsymbol{\eta}$ is positive on parts of $\boldsymbol{C}^{\sigma}$, this can be achieved by Ljapounoffs Theorem and the Inheritance Lemma in [6]. Note that the Density Lemma (Lemma 4.1 in [6] ) ensures

$$
\boldsymbol{\vartheta}^{(\rho)}\left(S^{\rho}\right)=\frac{\boldsymbol{\eta}\left(S^{\rho}\right)}{c_{\rho}} \leq \boldsymbol{\lambda}^{\rho}\left(S^{\rho}\right)=\boldsymbol{v}\left(R_{(\tau)}\right)
$$

for $\rho \in \boldsymbol{K}_{+}, \tau \in \boldsymbol{K}_{-}$.

Finally, we choose some fixed $\bar{\tau} \in \boldsymbol{K}_{-}$and put for $\rho \in \boldsymbol{K}_{0}$

$$
S^{\rho}:=R_{(\bar{\tau})}^{\rho} .
$$

This way we have now indeed defined a set

$$
S:=S^{1} \cup S^{2} \ldots \cup S^{r}
$$

satisfying

$$
\boldsymbol{v}(S)=\min \left\{\boldsymbol{\lambda}^{\rho}\left(S^{\rho}\right)\right\}=\boldsymbol{v}\left(R_{(\tau)}\right) \quad\left(\tau \in \boldsymbol{K}_{-}\right) .
$$

This holds true as the quantities in (3.5) and (3.8) are all equal.

$4^{\text {th }}$ STEP :

Now we prove that $\boldsymbol{\vartheta}$ exceeds $\boldsymbol{\eta}$ on $S$.

First of all consider $\tau \in \boldsymbol{K}_{-}$. We have

$$
\boldsymbol{\vartheta}_{\mid S^{\tau}}=\boldsymbol{\vartheta}_{\mid R_{(\tau)}^{\tau}} \geq c_{\tau} \boldsymbol{\vartheta}_{\mid R_{(\tau)}^{\tau}}^{\tau}>\boldsymbol{\eta}_{\mid R_{(\tau)}^{\tau}}=\boldsymbol{\eta}_{\mid} S^{\tau}
$$

by (3.3).

Next, consider $\rho \in \boldsymbol{K}_{+}$. For $\bar{\tau} \in \boldsymbol{K}_{\text {- we have }}$

$$
\boldsymbol{\vartheta}:=\sum_{\sigma \in \boldsymbol{K}_{+} \cup \boldsymbol{K}_{-}} c_{\sigma} \boldsymbol{\vartheta}^{(\sigma)} \geq c_{\rho} \boldsymbol{\vartheta}^{\rho}+c_{\bar{\tau}} \boldsymbol{\vartheta}^{\bar{\tau}}
$$

Recall that $\boldsymbol{\vartheta}^{(\bar{\tau})} \in \mathcal{S}$ is positive on $R_{(\tau)}^{\rho} \subseteq \boldsymbol{C}^{\rho}$ (see (3.6)). Also, $\boldsymbol{\eta} \in \mathcal{S}$ is positive somewhere on $\boldsymbol{C}^{\rho}$. Hence, by the Support Theorem(4.6 of [6]) both 
have the same carrier inside of $\boldsymbol{C}^{\rho}$ which necessarily includes $S^{\rho}$. Therefore, (3.12) may be continued on $S^{\rho}$ to imply

$$
\boldsymbol{\vartheta} \geq c_{\rho} \boldsymbol{\vartheta}^{\rho}+c_{\bar{\tau}} \boldsymbol{\vartheta}^{\bar{\tau}}=\boldsymbol{\eta}+c_{\bar{\tau}} \boldsymbol{\vartheta}^{\bar{\tau}}>\boldsymbol{\eta} \quad \text { on } S^{\rho}
$$

Finally, we have for $\rho \in \boldsymbol{K}_{0}$

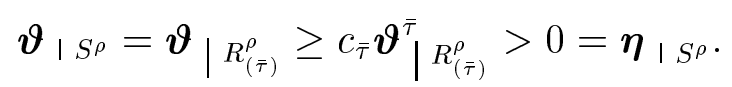

\section{$5^{\text {th }}$ STEP :}

Combining (3.14), (3.13), and (3.11), we obtain

$$
\vartheta>\boldsymbol{\eta} \text { on } \mathrm{S} \text {. }
$$

Now observe that

$$
\begin{aligned}
& \left.\boldsymbol{v}(S)=\sum_{\sigma \in \boldsymbol{K}_{+} \cup \boldsymbol{K}_{-}} c_{\sigma} \boldsymbol{v}(S)\right) \\
& =\sum_{\sigma \in \boldsymbol{K}_{+}} c_{\sigma} \boldsymbol{v}\left(R_{(\bar{\tau})}\right)+\sum_{\tau \in \boldsymbol{K}_{-}} c_{\tau} \boldsymbol{v}\left(R_{(\tau)}\right) \\
& \geq \sum_{\sigma \in \boldsymbol{K}_{+}} \boldsymbol{\eta}\left(S^{\sigma}\right)+\sum_{\tau \in \boldsymbol{K}_{-}} c_{\tau} \boldsymbol{\vartheta}^{\tau}\left(R_{(\tau)}\right) \quad \text { by }(3.9),(3.4) \\
& >\quad \sum_{\sigma \in \boldsymbol{K}_{+}} \boldsymbol{\eta}\left(S^{\sigma}\right)+\sum_{\tau \in \boldsymbol{K}_{-}} \boldsymbol{\eta}\left(R_{(\tau)}\right) \quad \text { by (3.3) } \\
& \geq \quad \sum_{\sigma \in \boldsymbol{K}_{+}} \boldsymbol{\eta}\left(S^{\sigma}\right)+\sum_{\tau \in \boldsymbol{K}_{-}} \boldsymbol{\eta}\left(S^{\tau}\right) \quad \text { by }(3.7) \\
& \geq \boldsymbol{\eta}\left(\bigcup_{\sigma \in \boldsymbol{K}_{+} \cup \boldsymbol{K}_{-}} S^{\sigma}\right) \\
& \geq \boldsymbol{\eta}(S) \text {. }
\end{aligned}
$$

Hence, for sufficiently small $\varepsilon>0$ the imputation

$$
\boldsymbol{\vartheta}^{\varepsilon}:=(1-\varepsilon) \boldsymbol{\eta}+\varepsilon \boldsymbol{\vartheta} \in \mathcal{S}
$$

exceeds $\boldsymbol{\eta}$ on $\mathrm{S}$ and still satisfies $\boldsymbol{\vartheta}^{\varepsilon}(S)<\boldsymbol{v}(S)$, that is, yields

$$
\boldsymbol{\vartheta}^{\varepsilon} \operatorname{dom}_{S} \boldsymbol{\eta}
$$

This proves that the assumption made at the beginning of the $2^{\text {nd }} S T E P$ leads to a contradiction, i.e., we have $\boldsymbol{K}_{-}=\emptyset$.

$\mathbf{6}^{\text {th }}$ STEP : Now,

$$
\boldsymbol{\eta}=\sum_{\sigma \in \boldsymbol{K}_{+}} c_{\sigma} \boldsymbol{\vartheta}^{(\sigma)}
$$


is a convex combination of elements of $\mathcal{S}$ that are concentrated on the carriers $\boldsymbol{C}^{\rho}\left(\rho \in \boldsymbol{K}_{+}\right)$. Necessarily, for every $\rho=1, \ldots, r$ there is an element of $\mathcal{S}$ that is positive on $\boldsymbol{C}^{\rho}$. By the above procedure, we find for every $\rho$ an element of $\mathcal{S}$ that is concentrated on $\boldsymbol{C}^{\rho}$. Hence, by Corollary 4.2. of [6], the Theorem is verified.

q.e.d.

Remark 3.2. Theorem 6.1. of [6] can be improved upon accordingly. That is, if $\mathcal{S}^{0}$ is a convex vNM-Stable Set for a linear production game constructed by orthogonal uniform distributions on a finite player set, then $\mathcal{S}^{0}$ is standard. For the proof, we do not have to assume that $\mathcal{S}^{0}$ is polyhedral. Of course, the reverse statement asserting that any standard set is a vNM-solution prevails in the finite case with uniform distributions as previously.

\section{References}

[1] Billera, L. J. And RaAnan, J.: Cores of Non-atomic Linear Production Games. Mathematics of Operations Research 6, (1981), pp. $420-423$.

[2] Einy, E:, Holzman, R., Monderer, D., And Shitovitz, B.: Core and Stable Sets of Large Games Arising in Economics. Journal of Economic Theory 68, (1996), pp $200-211$.

[3] HaRT, S.: Formation of Cartels in Large Markets. Journal of Economic Theory 7, (1974), pp. $453-466$.

[4] Shellshear, E.: vNM-Stable Sets for Linear Production Games with Orthogonal Good Distributions . Diploma Thesis, (2005), Institute of Mathematical Economics, University of Bielefeld.

[5] LuCas, W. F.: Von Neumann-Morgenstern Stable Sets. Handbook of Game Theory with Economic Applications, Vol. 1, Chapter 17, R. J. Aumann and S. Hart Eds., (1992), pp. 543 - 590.

[6] Rosenmüller, J., And Shitovitz, B: A Characterization of vNMStable Sets for Linear Production Games. International Journal of Game Theory 29, (2000), pp. 39 - 61.

[7] von Neumann, John and Morgenstern, Oskar: Theory of Games and Economic Behavior. Princeton University Press (1944, 1947, 1953), 641 pp. SBN 691-04183-0 